\title{
Heavy flavour spectroscopy at LHCb
}

\author{
Raphael MÄRKI* on behalf of the LHCb collaboration \\ Ecole Polytechnique Fédérale de Lausanne (EPFL) \\ E-mail: raphael.marki@epfl.ch
}

The LHCb experiment at CERN's Large Hadron Collider is dedicated to bottom and charm physics. The first $1.0 \mathrm{fb}^{-1}$ of $p p$ collisions collected during the 2011 run allows the exploration of the $b$-baryon sector with unprecedented precision. We present the first observation of $\Lambda_{b}^{*}$ baryons and measurements of the $\Xi_{b}^{-}$and $\Omega_{b}^{-}$baryon masses. In addition we present measurements of the properties of the $D_{s 1}^{*}(2700)^{+}$and $D_{s J}^{*}(2860)^{+}$mesons.

36th International Conference on High Energy Physics

July 4-11, 2012

Melbourne, Australia

${ }^{*}$ Speaker. 


\section{Introduction}

The measurement of the properties of the heavy-flavoured hadrons is essential to test both the heavy quark effective theory (HQET) and QCD sum rules. This report discusses the new observation of two excited beauty baryons, the measurement of the strange $b$-baryon masses, and a study of the $D_{s 1}^{*}(2700)^{+}$and $D_{s J}^{*}(2860)^{+}$mesons by the LHCb collaboration.

\section{Momentum scale calibration}

High precision mass measurements, especially for decays with large energy transfer, require a precise momentum measurement for each track. Therefore the absolute scale of the measured momentum in a detector, called momentum scale, needs to be calibrated. At LHCb, the momentum scale is calibrated using the well known $J / \psi$ resonance as reference. A correction is calculated based on the measured $J / \psi$ mass and applied to all reconstructed tracks. Other well known resonances are reconstructed using this procedure and the mass differences with repect to the known values are used to estimate the remaining momentum scale bias, called $\alpha$ (the factor by which the momentum of each track has to be multiplied to change the measured mass to the known value). The value of $\alpha$ is less than $0.02 \%$ for most decays, hence $0.02 \%$ is used as an estimate for the systematic uncertainty on the momentum scale and then propagated to an uncertainty on the measured mass.

To ensure its stability, this calibration is repeated for every period with different experimental conditions. During such a period, the measured $J / \psi$ mass has been demonstrated to stay well within the uncertainty coming from $\alpha$ [1].

\section{Beauty baryons}

Heavy baryons have many degrees of freedom compared to mesons. Baryons with a single heavy quark can be described as a system of a heavy quark and a light diquark. Even without considering orbital excitations, there is a large number of states with different quark content and spin configurations.

In the charm sector, many states, including orbital (and/or radial) excitations, have been observed at the $B$ factories. In contrast, the beauty-baryon sector is much less well studied. Until recently, only some of the ground states had been observed experimentally. In recent years, however, the Tevatron and LHC experiments have reported new observations of beauty-baryon states.

Large and clean samples of weakly decaying ground-state baryons are essential in order to study the strong decay of excited beauty baryons. The $\Lambda_{b}^{0}$ baryons are produced copiously in the hadronic environment. The CDF experiment reconstructs a signal of 19'300 $\Lambda_{b}^{0} \rightarrow \Lambda_{c}^{+} \pi$ decays with a signal-to-background ratio of around 1.9 [2] in their $6 \mathrm{fb}^{-1}$ data sample of $p \bar{p}$ collisions at $1.96 \mathrm{TeV}$. A recent analysis of LHCb [3] based on $1 \mathrm{fb}^{-1}$ of $p p$ collisions with $\sqrt{s}=7 \mathrm{TeV}$ uses a sample of $70^{\prime} 540 \pm 330 \Lambda_{b}^{0}$ reconstructed in the same final state with a signal-to-background ratio of 11 .

The ground state of the strange and doubly-strange $b$ baryons, $\Xi_{b}^{-}$and $\Omega_{b}^{-}$, have been studied previously by D0 [4, 5] and CDF [6]. 

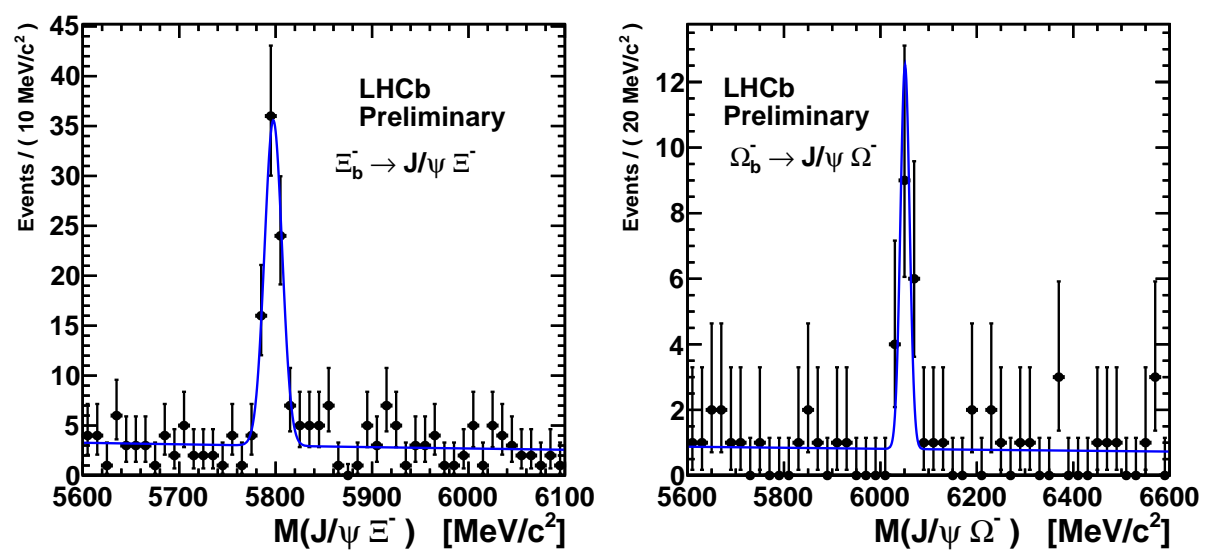

Figure 1: Invariant mass distributions of $\Xi_{b}^{-} \rightarrow J / \psi \Xi^{-}$(left) and $\Omega_{b}^{-} \rightarrow J / \psi \Omega^{-}$(right) candidates.

While the two collaborations obtain $\Xi_{b}^{-}$mass measurements compatible with each other, their $\Omega_{b}^{-}$mass measurements are inconsistent and differ by more than $100 \mathrm{MeV} / c^{2}$. This corresponds to an inconsistency of more than 6 standard deviations according to the quoted uncertainties of the measurements. LHCb has performed a study of the $\Xi_{b}^{-}$and $\Omega_{b}^{-}$states with a $576 \mathrm{pb}^{-1}$ data sample at $\sqrt{s}=7 \mathrm{TeV}$ [7]. The $\Xi_{b}^{-}$baryons are reconstructed in the decay chain $\Xi_{b}^{-} \rightarrow J / \psi \Xi^{-}$, $\Xi^{-} \rightarrow \Lambda \pi^{-}, \Lambda \rightarrow p \pi^{-}$; a signal of $72.2 \pm 9.4$ events is observed. Similarly, 13.9 ${ }_{-3.8}^{+4.5}$ events are observed in the decay $\Omega_{b}^{-} \rightarrow J / \psi \Omega^{-}, \Omega^{-} \rightarrow \Lambda K^{-}, \Lambda \rightarrow p \pi^{-}$with a significance above 5 standard deviations. The invariant mass distributions of these states are shown in Fig. 1 and their masses measured to be

$$
\begin{aligned}
& M\left(\Xi_{b}^{-}\right)=5796.5 \pm 1.2_{\text {stat }} \pm 1.2_{\text {syst }} \mathrm{MeV} / c^{2} \\
& M\left(\Omega_{b}^{-}\right)=6050.3 \pm 4.5_{\text {stat }} \pm 2.2_{\text {syst }} \mathrm{MeV} / c^{2}
\end{aligned}
$$

The $\Omega_{b}^{-}$result is in a good agreement with the CDF measurement [6], but in contradiction with the D0 measurement [5].

The LHCb collaboration has also observed a hint of $\Xi_{b}^{0}$ production in the study of the $D^{0} p K^{-}$ final state with a sample of $330 \mathrm{pb}^{-1}$, corresponding to $27 \pm 10$ events with a significance of 2.6 standard deviations [8]. The invariant mass distribution is shown in Fig. 2 and the $\Xi_{b}^{0}$ mass is measured to be

$$
M\left(\Xi_{b}^{0}\right)=5802.0 \pm 5.5_{\text {stat }} \pm 1.7_{\text {syst }} \mathrm{MeV} / c^{2} .
$$

No experimental evidence of the orbitally-excited beauty baryons was available until recently. The quark model predicts two $L=1$ excitations of the $\Lambda_{b}^{0}$ baryon with $J^{P}=1 / 2^{-}$and $3 / 2^{-}$. Both should decay to $\Lambda_{b}^{0} \pi^{+} \pi^{-}$or $\Lambda_{b}^{0} \gamma$, depending on their masses. Most theoretical predictions give masses above the $\Lambda_{b}^{0} \pi^{+} \pi^{-}$threshold at $5900 \mathrm{MeV} / c^{2}$, but below the $\Sigma_{b} \pi$ threshold.

The first observation of orbitally-excited beauty baryons has been made by LHCb [3] in the final state $\Lambda_{b}^{0} \pi^{+} \pi^{-}$using $1 \mathrm{fb}^{-1}$ of data at $\sqrt{s}=7 \mathrm{TeV}$. The $\Lambda_{b}^{0}$ is reconstructed in the $\Lambda_{c}^{+} \pi^{-}, \Lambda_{c} \rightarrow$ $p K^{-} \pi^{+}$final state and is combined with a pair of pion tracks coming from the primary interaction vertex. A kinematic fit with $\Lambda_{b}^{0}$ and $\Lambda_{c}^{+}$mass constraints is performed to improve the invariant mass 


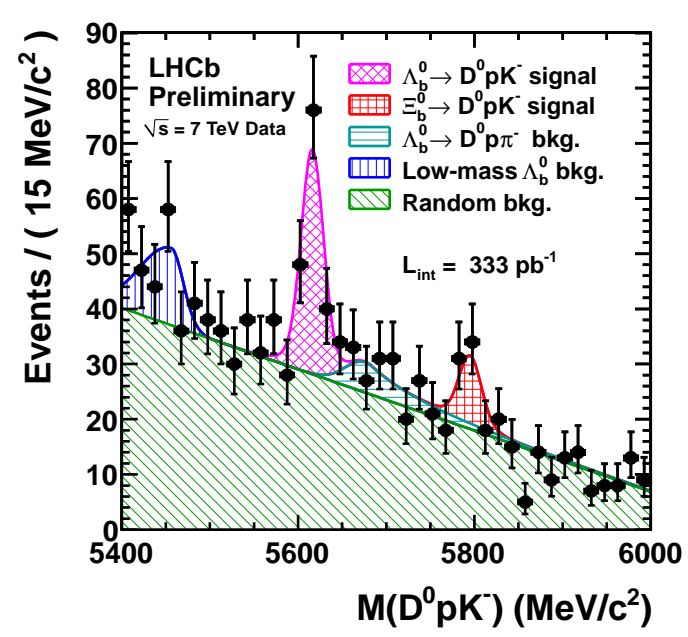

Figure 2: Invariant mass distribution for $D^{0} p K^{-}$combinations.

resolution. The right-sign $\Lambda_{b}^{0} \pi^{+} \pi^{-}$and wrong-sign $\Lambda_{b}^{0} \pi^{ \pm} \pi^{ \pm}$distributions are shown in Fig. 3. In the right-sign spectrum, two narrow peaks are evident at masses around $5912 \mathrm{MeV} / \mathrm{c}^{2}$ and $5920 \mathrm{MeV} / c^{2}$, with signal yields of $16.4 \pm 4.7$ and $49.5 \pm 7.9$ events, respectively. The proximity of the kinematic threshold results in an excellent invariant mass resolution of $0.2-0.3 \mathrm{MeV} / c^{2}$. The fit of the spectrum yields the following masses for the two states:

$$
\begin{aligned}
& M_{\Lambda_{b}^{* 0}(5912)}=5911.95 \pm 0.12_{\text {stat }} \pm 0.03_{\text {syst }} \pm 0.66_{\Lambda_{b}^{0} \text { mass }} \mathrm{MeV} / c^{2}, \\
& M_{\Lambda_{b}^{* 0}(5920)}=5919.76 \pm 0.07_{\text {stat }} \pm 0.02_{\text {syst }} \pm 0.66_{\Lambda_{b}^{0} \text { mass }} \mathrm{MeV} / c^{2},
\end{aligned}
$$

where the third error is induced by the uncertainty on the $\Lambda_{b}^{0}$ mass being constraint in the $\Lambda_{b}^{* 0}$ reconstruction. The significances of the observation (including systematic effects and the trial factor in the mass range 5900-5950 MeV/ $\mathrm{c}^{2}$ ) are 4.9 and 10.1 standard deviations, respectively. Upper limits at $95 \%$ confidence level on the decay widths of the two states are also obtained as

$$
\begin{aligned}
& \Gamma_{\Lambda_{b}^{* 0}(5912)}<0.82 \mathrm{MeV} / c^{2}, \\
& \Gamma_{\Lambda_{b}^{* 0}(5920)}<0.71 \mathrm{MeV} / c^{2} .
\end{aligned}
$$

\section{Charm mesons}

Charm mesons are bound states of a heavy charm quark of spin $\overrightarrow{S_{Q}}(Q=c)$ and a light quark of spin $\overrightarrow{s_{q}}(q=u, d, s)$, characterized by their orbital angular momentum $\vec{L}$ and total spin $\vec{J}=\overrightarrow{S_{Q}}+\overrightarrow{j_{q}}$, where $\overrightarrow{j_{q}}=\overrightarrow{s_{q}}+\vec{L}$. The lowest-lying states have $L=0$ and are denoted as $D\left(D^{*}\right)$ when $J^{P}=0^{-}\left(1^{-}\right)$. The first orbital excitations $(L=1)$ have either $j_{q}=1 / 2\left(D_{0}^{*}, D_{1}^{\prime}\right.$ with $\left.J^{P}=0^{+}, 1^{+}\right)$or $j_{q}=3 / 2$ $\left(D_{1}, D_{2}^{*}\right.$ with $\left.J^{P}=1^{+}, 2^{+}\right)$.

While the spectroscopy of the $D^{0}$ and $D^{+}$states follows the predictions of HQET, the charmstrange meson system is a more interesting case: the $D_{s 0}^{*}(2317)^{+}$and $D_{s 1}(2460)^{+}$states observed 

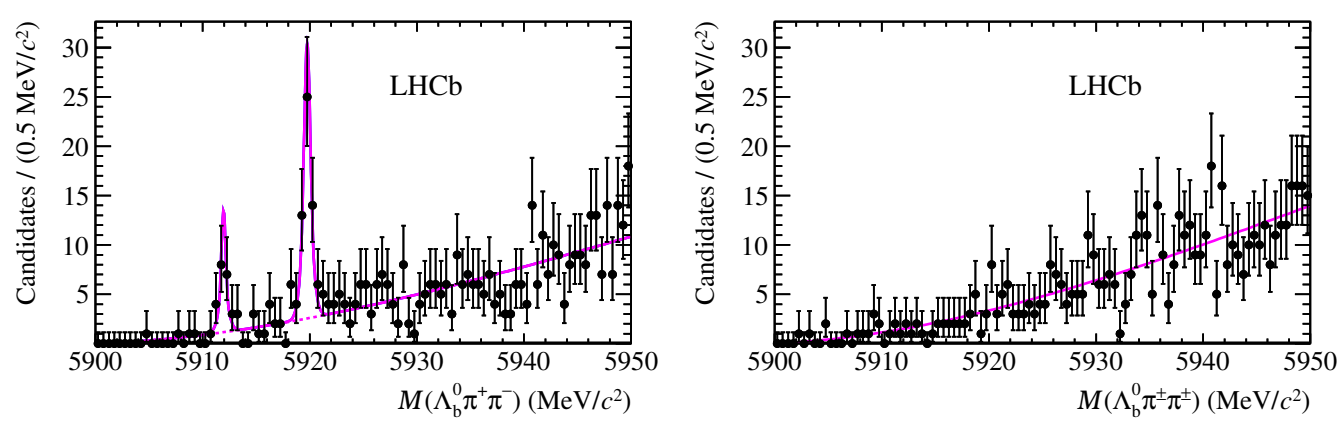

Figure 3: Invariant mass distributions of $\Lambda_{b}^{0} \pi^{+} \pi^{-}$(right-sign, left) and $\Lambda_{b}^{0} \pi^{ \pm} \pi^{ \pm}$(wrong-sign, right) combinations.

by BABAR [9] and Belle [10] almost a decade ago have masses considerably smaller than the HQET predictions. More recently, the $D_{s 1}^{*}(2700)^{+}$and $D_{s J}^{*}(2860)^{+}$states were observed $[11,12]$.

The existence of these latter two states has recently been confirmed by the LHCb collaboration [13] using a sample of $p p$ collisions corresponding to $1 \mathrm{fb}^{-1}$ taken in 2011 at $\sqrt{s}=7 \mathrm{TeV}$. The combinations $D^{+} K_{S}^{0}$ and $D^{0} K^{+}$are studied, where the $D$ and $K$ mesons are required to originate from the $p p$ interaction vertex. The corresponding invariant mass distributions are shown in Fig. 4. A combined fit to both spectra is performed, where the signals are modeled by Breit-Wigner shapes with Blatt-Weisskopf form factors, and the background from random $D K$ combinations is represented by polynomial shapes. The most prominent peak in the distributions is $D_{s 2}^{*}(2573)^{+}$, while the contributions from $D_{s 1}^{*}(2700)^{+}$and $D_{s J}^{*}(2860)^{+}$are also clearly visible. The parameters of the $D_{s 1}^{*}(2700)^{+}$and $D_{s J}^{*}(2860)^{+}$states obtained from the fit are ${ }^{1}$

$$
\begin{aligned}
M\left(D_{s 1}^{*}(2700)^{+}\right) & =\left(2709.4 \pm 1.9_{\text {stat }} \pm 4.5_{\text {syst }}\right) \mathrm{MeV} / c^{2}, \\
\Gamma\left(D_{s 1}^{*}(2700)^{+}\right) & =\left(121.7 \pm 7.3_{\text {stat }} \pm 12.1_{\text {syst }}\right) \mathrm{MeV} / c^{2}, \\
M\left(D_{s J}^{*}(2860)^{+}\right) & =\left(2866.7 \pm 1.0_{\text {stat }} \pm 6.3_{\text {syst }}\right) \mathrm{MeV} / c^{2}, \\
\Gamma\left(D_{s J}^{*}(2860)^{+}\right) & =\left(64.5 \pm 3.2_{\text {stat }} \pm 6.6_{\text {syst }}\right) \mathrm{MeV} / c^{2} .
\end{aligned}
$$

The largest contributions to the systematic errors are due to the signal and background models and the uncertainty in the invariant mass resolution. The results are consistent with the measurements performed by BABAR [14] and Belle [12]. There is no evidence in the $D K$ spectra for a state with a mass around $3040 \mathrm{MeV} / c^{2}$ corresponding to that reported by BABAR in the $D^{*} K$ spectrum [14].

\section{Summary}

Numerous new results in charm and beauty hadron spectroscopy have appeared in the recent two years. After the start of LHC, the LHCb experiment has not only confirmed some of the observations made previously by the Tevatron and $B$ factories (such as the observation of strange $b$ baryons and $D_{s 1}^{*}(2700)^{+}, D_{s J}^{*}(2860)^{+}$and $B_{(s)}^{* * 0}$ mesons), but has also observed new states: orbitally excited $\Lambda_{b}^{0}$ baryons. The masses of the new beauty hadrons are consistent with the theoretical predictions. The quantum numbers and other properties of many states remain to be determined.

\footnotetext{
${ }^{1}$ Shortly after the conference, this analysis has been updated and published in a journal with the finalized values [13].
} 

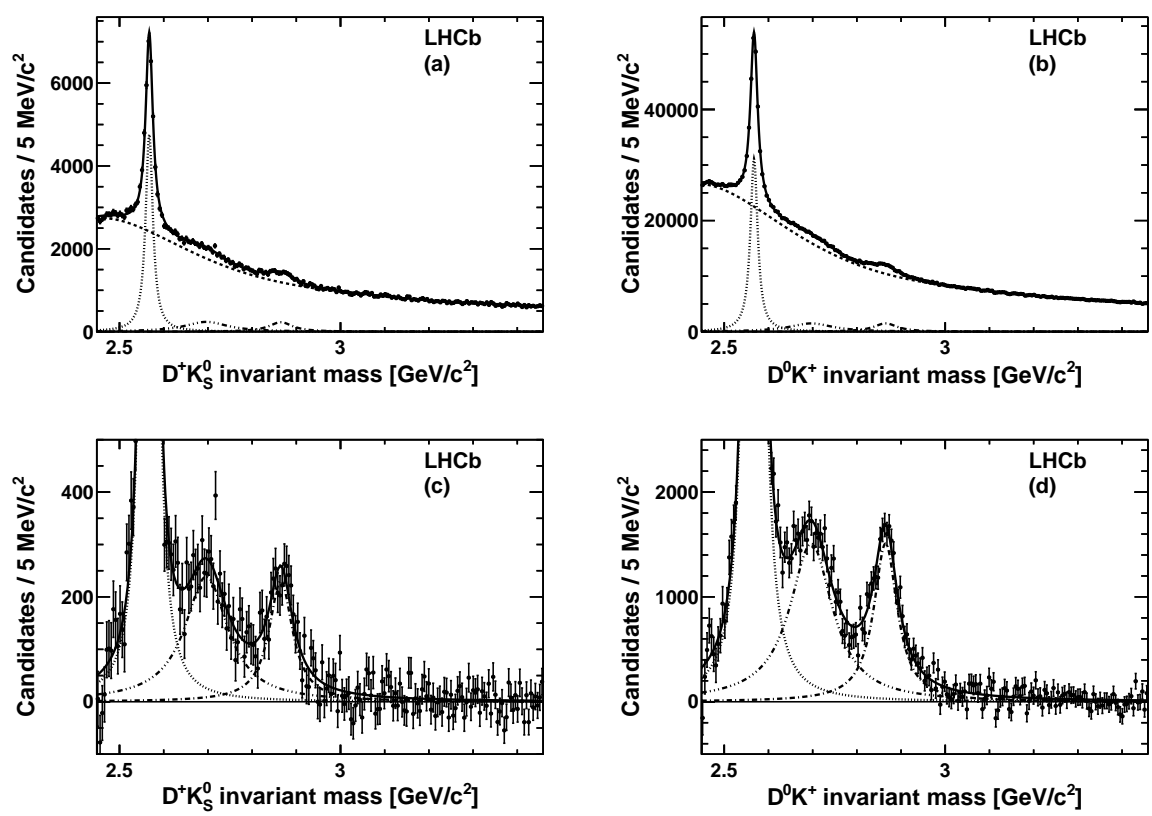

Figure 4: Invariant mass distributions (points) for (a) $D^{+} K_{S}^{0}$ and (b) $D^{0} K^{+}$. We show the total simultaneous probability density function (solid line), the $D_{s 2}^{*}(2573)^{+}$(fine dotted line), $D_{s 1}^{*}(2700)^{+}$(dot-dot-dot dashed line), $D_{S J}^{*}(2860)^{+}$(dot dashed line) and background contribution (dashed line). Invariant mass distributions after combinatorial background subtraction are shown for (c) $D^{+} K_{S}^{0}$ and (d) $D^{0} K^{+}$, where the vertical scales are truncated to show the $D_{s 1}^{*}(2700)^{+}$and $D_{s J}^{*}(2860)^{+}$signals more clearly.

\section{References}

[1] R. Aaij et al. [LHCb Collaboration], Phys. Lett. B 708 (2012) 241-248 [arXiv:1112.4896].

[2] T. Aaltonen et al. [CDF Collaboration], Phys. Rev. D 85 (2012) 092011 [arXiv:1112.2808].

[3] R. Aaij et al. [LHCb Collaboration], Phys. Rev. Lett. 109 (2012) 172003 [arXiv:1205.3452].

[4] V. M. Abazov et al. [D0 Collaboration], Phys. Rev. Lett. 99 (2007) 052001 [arXiv:0706.1690].

[5] V. M. Abazov et al. [D0 Collaboration], Phys. Rev. Lett. 101 (2008) 232002 [arXiv:0808.4142].

[6] T. Aaltonen et al. [CDF Collaboration], Phys. Rev. D 80 (2009) 072003 [arXiv:0905.3123].

[7] LHCb Collaboration, LHCb-CONF-2011-060.

[8] LHCb Collaboration, LHCb-CONF-2011-036.

[9] B. Aubert et al. [BABAR Collaboration], Phys. Rev. Lett. 90 (2003) 242001 [hep-ex/0304021].

[10] P. Krokovny et al. [Belle Collaboration], Phys. Rev. Lett. 91 (2003) 262002 [hep-ex/0308019].

[11] B. Aubert et al. [BABAR Collaboration], Phys. Rev. Lett. 97 (2006) 222001 [hep-ex/0607082].

[12] J. Brodzicka et al. [Belle Collaboration], Phys. Rev. Lett. 100 (2008) 092001 [arXiv:0707.3491].

[13] R. Aaij et al. [LHCb Collaboration], JHEP 10 (2012) 151 [arXiv:1207.6016].

[14] B. Aubert et al. [BABAR Collaboration], Phys. Rev. D 80 (2009) 092003 [arXiv:0908.0806]. 